\title{
RACIONALISMO MODAL, NATURALISMO Y ESCEPTICISMO EMPIRISTA ${ }^{1}$
}

\author{
MODAL RATIONALISM, NATURALISM \\ AND EMPIRICIST SKEPTICISM
}

\author{
Rafael Miranda-Rojas* \\ Universidad Católica del Maule \\ Talca-Chile \\ Recibido febrero de 2020/Received February, 2020 \\ Aceptado octubre de 2020/Accepted October, 2020
}

\begin{abstract}
RESUMEN
Recientemente Machery (2017) ha desarrollado una crítica a la práctica filosófica fundada en casos hipotéticos, el denominado método de casos (method of cases). Una vía para cuestionar esta práctica y el rol de las intuiciones en el mismo es el denominado argumento desde la no confiabilidad (unreliability). De esta no confiabilidad se sigue una conclusión escéptica respecto de los análisis modales característicos de ciertos argumentos filosóficos: la exigencia de suspensión de juicio ante los casos filosóficos. Esta postura conduce a sostener una inmodestia filosófica respecto de los límites epistémicos que no permitirían, por ejemplo, solucionar los desacuerdos perennes presentes en el ámbito filosófico. Ello deriva a su vez en dos consecuencias cuestionables de la argumentación filosófica, según Machery: dogmatismo (dogmatism) o insularidad (parochialism). Mi objetivo en este escrito es analizar qué noción confiabilista está en juego en esta postura experimental negativa (negative Xphi) desde la propuesta desarrollada por Goldman (2013), y evaluar la validez de una postura escéptica empirista, considerando el escepticismo como una postura prima facie especulativa y, por tanto, no contrastable empíricamente. Si lo anterior es correcto, observo que existe un problema en una postura experimental escéptica.
\end{abstract}

Palabras Clave: Intuición, Filosofía Experimental, Racionalismo, Confiabilidad, Escepticismo.

\begin{abstract}
Recently, Machery (2017) has developed a critique of philosophical practice based on hypothetical cases, the so-called method of cases. One way to question this practice and the role of intuitions is the so-called argument from unreliability. From this non-reliability a skeptical conclusion follows regarding the modal analyzes characteristic of certain philosophical arguments: the requirement of suspension of judgment concerning philosophical cases. This posit leads to maintain a philosophical immodesty regarding the epistemic limits that would not allow, for example, to solve the perennial disagreements present in the philosophical field. This in turn results in two questionable consequences of philosophical argumentation, according to Machery: dogmatism (dogmatism) and / or parochialism. My objective in this paper is to analyze what reliability notion is at stake in this negative experimental stance (negative Xphi) from the proposal developed by Goldman (2013), and to evaluate the validity of an empiricist skeptical stance, considering skepticism as prima facie speculative and, therefore, not empirically testable. If this is correct, there is a problem in a skeptical experimental position.
\end{abstract}

Key Words: Intuition, Experimental Philosophy, Rationalism, Reliability, Skepticism. 


\section{LOS ROLES EPISTÉMICOS DE UNA INTUICIÓN}

En el desarrollo de la argumentación filosófica, las así denominadas intuiciones racionales han tenido una relevancia que actualmente es foco de una creciente discusión centrada en su legitimidad y su potencial ausencia de contrastación empírica. Es por tanto una arista central del debate racionalismo-naturalismo ${ }^{2}$, y la discusión evidencial subyacente ¿Qué se entiende por evidencia? ¿Es válido sostener que hay evidencia a priori? ¿Es la intuición un caso de evidencia a priori o, en su defecto, una vía confiable de acceso epistémico a algún ítem de evidencia a priori? Estas son preguntas que prima facie guían el debate actual entre la así denominada filosofía de sillón (armchair philosophy) y la filosofía experimental (Xphi). El objetivo en este escrito es analizar qué noción confiabilista desarrolla la postura experimental negativa (negative Xphi) siguiendo a Goldman (2013). Luego, evaluar la validez de una postura escéptica empirista, considerando el escepticismo como una postura prima facie especulativa (no contrastable empíricamente). A partir de lo anterior, se releva una propuesta racionalista naturalista moderada, que permita relevar el rol propedéutico de ciertos insumos modales, especialmente las intuiciones y las situaciones contrafácticas presentes en diversos experimentos mentales.

Las evidencias empíricas contra las intuiciones desarrolladas desde la investigación experimental han planteado como problema central la confiabilidad (reliability) que estas tienen como antecedente que guía ciertas argumentaciones filosóficas, y sus conclusiones. Sostienen en general que la variabilidad de las mismas entre culturas (cross cultural variability) sería un punto en contra para el uso que la filosofía le otorga en su construcción teórica a partir de casos hipotéticos, por esta razón no contrastables (inicialmente) empíricamente. Lo anterior es lo que se propone denominar variabilidad intuicional ${ }^{3}$, el que las intuiciones varíen entre culturas, por ejemplo. El punto en discusión lo resume claramente Goldman al explicitar:

\footnotetext{
"Consulting intuitions about imaginary cases is a paradigm of 'armchair' philosophy. Whatever else is meant by an 'armchair' method, it is certainly intended to contrast with the method of empirical science, which uses 'real' experimentation and observation
}

rather than reflection on hypothetical examples. Philosophical naturalists tend to distrust armchair methods. They embrace empirical science as the paradigm of sound methodology. Philosophy should not be dragged into epistemological disrepute by utilizing suspect faculties or procedures like intuition. Traditionalists reply that intuition is a perfectly sound faculty; it is just an $a$ priori faculty rather than an empirical one. Rationalists are happy to defend intuition, which they see as closely allied to, if not identical with, the faculty of reason. Hence the sharp split, at least in initial instincts, between traditionalists and philosophical naturalists" (Goldman 2013, 12).

En el desarrollo de la argumentación filosófica, las así denominadas intuiciones racionales han tenido una relevancia que actualmente es foco de una creciente discusión centrada en su legitimidad y su potencial ausencia de contrastación empírica. Es por tanto una arista central del debate racionalismo-naturalismo. De este modo, un supuesto de la discusión respecto del rol evidencial de las intuiciones sería que

i) La confiabilidad intuicional reside en entregar evidencia a favor o en contra de cierta postura específica ${ }^{4}$.

Goldman propone abordar este problema desde una distinción entre cuestiones de primer orden (first order question about evidence) y segundo orden (second order question about evidence), siendo el foco principal del primer caso la posibilidad de que haya evidencia a priori, no empírica, y el segundo caso qué justifica sostener que cierto ítem evidencial a priori (la intuición en este caso) sea efectivamente evidencia de p. Si las cuestiones de segundo orden derivan en una petitio principii (que lo que permita sostener el carácter evidencial de una intuición en este caso sea otra intuición, por ejemplo), es importante tener en vistas que es un problema que podría eventualmente extenderse a cualquier ítem evidencial; por ejemplo, que se requiere más evidencia empírica para justificar la validez de la evidencia empírica de primer orden. Este punto toca el cómo se calibra o evalúa la validez de la información a la que se accede mediante distintos accesos epistémicos. De todos modos, el foco de este escrito no es defender el rol evidencial de una intuición, y mencionar este punto tiene como 
objetivo visibilizar los límites de ciertos argumentos contra las intuiciones. A su vez, esto no equivale a descartar que cierta información empírica cumpla o pueda cumplir un rol de defeater de ítems de evidencia que se comprenden como a priori. En efecto, un análisis reciente realizado por Ichikawa (2014) sostiene el rol de undercutting defeater (derrotador debilitante) a cierta información empírica que muestre la falsedad de cierta intuición. En otras palabras, cuestionar que ciertas razones para creer $\mathrm{p}$, no cumplen el rol de indicador de verdad (truth indicator) de $\mathrm{p}$ : esas razones no serían razones a favor de que $\mathrm{S}$ crea $\mathrm{p}$, siendo las intuiciones un caso de estas razones. No es completamente claro que las conclusiones de la filosofía experimental negativa sean un caso de este tipo, o de los así denominados rebutting defeaters, entendiendo este como evidencia contra la creencia que p. Machery sostiene un compromiso con el confiabilismo que considera estos defeater, en vistas a evitar casos de conocimiento fácil (easy knowledge). Lo interesante es que, en los casos paradigmáticos de situaciones contrafácticas criticadas, son dos opciones las consideradas: p o no - p. El descarte de una de estas alternativas parecería otorgar no solo confiabilidad a las conclusiones confiabilistas, sino certeza:

"...theories that insist that epistemic achievement is compatible with unreliability... concur on the importance of reliability in the present case. Achievement requires doing substantially better than luck, and one has a chance out of two to get it right when the choice is between $p$ or not $p \ldots$... So, achievement in the present case requires reliability (Machery 2017, 97).

Este es un aspecto relevante que considera la postura pro intuicional desarrollada por Goldman (2013) desde una perspectiva no solipsista, lo que será analizado líneas abajo. Un desideratum de una potencial relación fructífera entre ambos es que ese potencial rol pudiese cumplirse a la inversa: que ciertos accesos a priori sean potenciales defeaters de información empírica que, por diversos motivos, no cumpliese ciertos criterios de adecuación. Por ejemplo, que la interpretación de un experimento y su ulterior argumentación sea sesgada y que, por tanto, esa argumentación no cumpla el rol evidencial que se pretende en dicha argumentación. Machery afirma que los juicios elaborados en la argumentación filosófica son artefactos cognitivos (cognitive artifacts) no confiables.

\begin{abstract}
“...judgments elicited by philosophical cases are often 'cognitive artifacts' Philosophers relying on these cases are like astronomers who would take instrumental artifacts at face value when they theorize about astronomical phenomena, or biologists who would take the deformations produced by microscopes for real phenomena" (Machery 2017, 90).
\end{abstract}

No lo son, pues estarían expuestos a lo que el autor denomina disturbing characteristics (características perturbadoras ${ }^{5}$ ) Pero ¿qué noción de confiabilidad o no confiabilidad es la que está en juego aquí? ¿Corresponde a la postura estándar de que una creencia es justificada y verdadera si es adquirida mediante un proceso confiable? Goldman analiza que, en vista a sostener algún rol evidencial de un juicio intuitivo, este puede analizarse desde la perspectiva de primer orden o la de segundo orden, correspondiendo la primera a evaluación a priori, la segunda a evaluación empírica o a posteriori. Siguiendo a Kelly (2008), comprende Goldman $(2013,14)$ como reliable indicator (indicador confiable) aquel ítem: in which $X$ is evidence for $Y$ if and only if $X$ is a (fairly) reliable sign, or indicator, of (the truth or existence of) $Y$. Esto se alinea con la comprensión clásica del confiabilismo, en la que el foco central de discusión es qué vías de acceso epistémico son confiables o conductoras de verdad (truth conduciveness) Afirmar que cierta vía no es confiable redunda en sostener, por tanto, que ese vínculo con algún ítem de conocimiento o justificación no es correcto, que no es el caso que dicha vía permita determinar como verdadera cierta creencia respecto a p. Los ejemplos usuales son por tanto los negativos, en los que parece evidente que un cierto acceso no puede ser comprendido como conductor de verdad, ni menos de conocimiento, la clarividencia (clairvoyance) como caso de acceso no confiable (BonJour $1980^{6}$ ). No es errado sostener que la lectura que tiene una postura experimental negativa de la metodología tradicional de análisis de casos hipotéticos es precisamente que algunos filósofos acceden a cierta información por medio de vías cuya justificación y confiabilidad no ha sido explicada. De hecho, Machery explicita el riesgo epistémico de no establecer una distinción entre accesos confiables y no confiables (entendiendo los juicios intuitivos como no confiables). 
"If judgments produced by an unreliable process were not severely deficient from an epistemic point of view, then choosing what to believe at random (by, e.g., throwing a coin to decide what to believe) or choosing on the basis of a process that works as designed but does not do better than a random process would result in epistemically appropriate or only moderately deficient beliefs" (Machery 2017, 97).

La no confiabilidad de los juicios intuitivos sería analogable a los casos estocásticos enunciados. Casos incuestionables de no conocimiento son útiles para comprender los alcances del criterio de confiabilidad, y a su vez de no confiabilidad. Siguiendo a Becker ${ }^{7}$, la principal característica de un confiabilismo referente al proceso de formación de creencias (process reliabilism) es que:

"S knows that $\mathrm{p}$ if and only if $\mathrm{S}$ believes that $p, p$ is true, and S's belief that $p$ is formed by a reliable process. A truth-conducive or reliable process is sometimes described as a belief-forming process that produces either mostly true beliefs or a high ratio of true to false beliefs".

Por su parte, la descripción del indicador confiable que explicita Goldman (2013, p. 13) corresponde a un análisis de estado de cosas (state of affairs) o hechos (facts), siendo estos los casos paradigmáticos de indicadores confiables de creencia:

"... there being 743 rings on the trunk of a given tree is (good) evidence for the proposition that the tree is 743 years old. That a column of mercury in a given thermometer is at the 70degree mark is (good) evidence that the ambient temperature is 70 degrees Fahrenheit. That a clock reads ' $4: 45$ ' may be (good) evidence that the current time at the clock's location is 4:45. In all of these cases the specified state of affairs constitutes evidence for the specified relatum because the indicated state of affairs is a rather reliable indicator of the truth of the proposition specified".

En cada ejemplo, confiabilidad no redunda en inmunidad al error o infalibilidad. Solo da cuenta del proceso usual que es viable sostener que $\mathrm{S}$ no comete un error epistémico al considerar como confiable la información obtenida por medio de ciertos procesos epistémicos. No obsta lo anterior que precisamente ejemplos como los explicitados sean sometidos a la crítica escéptica, como el caso Truetemp (Lehrer (1990) discutido inter alia ulteriormente en Swain, Alexander y Weinberg (2008)) respecto de la medición de temperatura y la posibilidad de conocimiento fácil (easy knowledge) si es el caso que un sujeto $S$ accede a esa información por procesos no confiables: hay una apelación intuitiva a no considerar casos de conocimiento por suerte como casos legítimos de conocimiento. La postura que dirige la reflexión respecto de las intuiciones es que estas son un caso de estado mental (mental state), y por esta razón la pregunta que se realiza es si este estado mental puede contar como evidencia. Lycan (1988) ya anticipaba la relevancia de distinguir por tanto entre entender el contenido del estado mental (intuited) o el estado mental en sí mismo (intuiting) como evidencia de p. Así, considerando la eventual relevancia del criterio seems true (parecer verdadero) y en línea con lo afirmado por Huemer (ver nota 2), afirma Goldman:

\begin{abstract}
"Seeming to see an apple on a nearby table may be a reliable indicator of there being an apple on a nearby table. Seeming to remember eating granola for breakfast may be a reliable indicator that one did eat granola for breakfast" (Goldman 2013, 14).
\end{abstract}

\section{ALCANCES DE LAS INTUICIONES EN EL DEBATE FILOSÓFICO MODAL}

La cuestión de fondo aquí es que, si bien hay proposiciones acerca de estados de cosas o hechos que parecen verdaderos sin serlo, también es correcto que los casos legítimamente verdaderos usualmente comparten ese criterio; hay excepciones, pero ellas generalmente radican en casos que requieren experticia previa, no descartando por tanto que quienes tengan esa experticia sí les parezca que cumplen con ese criterio. No debe sorprender, considerados los ejemplos previos, que la evidencia de lo intuitivo sea en estos casos de segundo orden, validando de ese modo que si S intuye $\mathrm{p}$, p es confiable. Goldman $(2013,21)$ destaca esta interpretación del juicio intuitivo como un caso de estado intencional que, de acuerdo con su propuesta de calificación intuicional, no determina el contenido de los denominados free-floating classifiers, siendo estos clasificadores de pertenencia o no pertenencia a cierto grupo evaluado intutivamente (no inferencialmente), y que incluye ítems relevantes en el ámbito filosófico como propiedades, universales, clases (kinds), inter alia. No son, por 
tanto, un caso de objeto intencional en el sentido que su rol de clasificadores supervenga a un individuo o a un grupo específico. No es completamente claro, no obstante, qué criterios allende la intuición determinan el juicio de pertenencia o no a cierto clasificador. Esto evitaría la demanda de ausencia de evidencia desde una postura confiabilista, en la que se descarta que cumplir con las condiciones

\section{$S$ cree $p$}

p es verdadero

sean condiciones suficientes para sostener que $S$ conoce $\mathrm{p}$, considerando el peso de contraejemplos en los que se cumplirían ambas condiciones, y sin embargo $\mathrm{S}$ no accede de modo confiable a p. Otro ejemplo paradigmático de este tipo es el fenómeno del sesgo de confirmación (confirmation bias), el del mero adivinar (guess), o el de creencia obstinada (willfull belief). La posición estándar descarta un nexo entre confiabilidad y verdad en casos en que $\mathrm{S}$ desea que $\mathrm{p}$, imagina que $\mathrm{p}$, teme que $\mathrm{p}$, inter alia (Goldman ibíd.)

Respecto de la incidencia de algunos sesgos (bias) Timothy Williamson $(2016,25)$ comete un error similar al referirse a quienes defienden una postura opuesta a la suya como mad-dog naturalists (perros rabiosos naturalistas). Esto, que podría entenderse como un mero uso estilístico, constituye el foco del debate de fondo del rol de la filosofía experimental y el rol que tienen las denominadas intuiciones filosóficas (philosophical intuitions) en el debate metodológico: el rol de los sesgos (biases) en la fundamentación de lo que un sujeto $\mathrm{S}$ cree. Previo a esta afirmación, Williamson intenta mostrar que el problema de la metodología filosófica no son los casos abordados por la filosofía de sillón (armchair philosophy), pues esos casos pueden ser replicables empíricamente en vista a evitar el problema del acceso a priori; al menos la mayoría de ellos, defiende el autor. Los así denominados perros rabiosos naturalistas corresponden a aquellos filósofos que defienden una postura experimental negativa: descartar la confiabilidad de las intuiciones filosóficas a todo evento, descartar por tanto su utilidad epistémica y, tal vez peor, afirmar irresponsabilidad epistémica de quienes confíen en ellas sin un contraste empírico de las mismas. Implícitamente se asume que estados intencionales como los enunciados líneas arriba no tienen relevancia específica en el estatuto epistémico de si es verdadero que p, su estatuto alético específico. Ello no parece extraño, si parece implausible sostener que se puede acceder a la verdad de $\mathrm{p}$ solo si S desea que $\mathrm{p}$ sea verdadero, pues ese estado intencional de S no contaría como evidencia a favor (o en contra) del valor de verdad de p. Sí sería extraño descartar el rol de conductor de verdad del estado mental en cuestión, un acceso epistémico válido. Goldman en rigor afirma la baja probabilidad (unlikely) de que estos estados mentales sean confiables en el sentido requerido ¿Por qué en el caso de intuir que p sería distinto? Goldman $(2013,14)$ enuncia:

\section{"....intuiting that p may be one of the mental states that is a reliable indicator of the truth of its contents, at least for a suitably delimited class of intuitings. If so, such intuitings would qualify as evidential states in the reliable- indicator sense".}

La explicación de por qué esto es así no es dada, pero sí sugiere que es el contenido del estado mental lo que permitiría sostener aquello. Sin embargo, si ese fuese el caso, el punto en cuestión no aborda la confiabilidad de lo intuido en tanto intuido, ello sería accidental al contenido proposicional per se. En otras palabras, el valor de verdad del contenido intuido no estaría justificado por el hecho de ser intuido y, por tanto, no se podría sostener como evidencia a favor de valor de verdad de $p$, el que $S$ intuya $p$ : intuir no sería un estado mental evidencial a favor (o en contra) de $\mathrm{p}$ :

\footnotetext{
"When a philosopher judges that a character in a Gettier case fails to know proposition $p$, the item of evidence isn't the philosopher's intuitive judgment; it is the (truth of) the proposition so judged, namely, that the character doesn't know p" (Ibíd.).
}

Williamson $(2007,2010)$ observa esta problemática potencial psicologización (psychologizing) de cierta evidencia, un foco de crítica a la postura experimental. El foco del análisis filosófico apoyado en situaciones contrafácticas o experimentos mentales no es otorgar evidencia intuitiva que permita sostener el estatuto epistémico confiable de esos casos. Allende de que no se resuelve que estatuto ontológico corresponde a este tipo de estados modales, no dando por establecido que pueden o deben analogarse con estado de cosas (state of affairs) o 
hechos (facts). En efecto, un antecedente relevante de un nexo entre contrastación empírica y el aporte de la evaluación contrafáctica lo constituye la condición de discriminación perceptual discutida en Goldman (1976, 786), cuyo ejemplo paradigmático es el del granero simulado (fake barn):

\begin{abstract}
"S has perceptual knowledge if and only if not only does his perceptual mechanism produce true belief, but there are no relevant counterfactual situations in which the same belief would be produced via an equivalent percept and in which the belief would be false".
\end{abstract}

Este criterio de contrastación contrafáctica (si se permite el uso de contrastación), si bien presenta diversos problemas (cómo evitar consecuencias escépticas respecto de que un sujeto $\mathrm{S}$ no conoce si no discrima diversos escenarios contrafácticos) sugiere también que la evidencia empírica requiere evaluación ulterior en vista a determinar un acceso epistémico confiable aunque falible.

La discusión entre la filosofía de sillón (armchair philosophy) y la postura experimental negativa (negative Xphi) no ha considerado ampliamente, salvo la importante excepción de Williamson (2007), la arista metafísica como eje central en el debate. Concerniente a la experticia filosófica subyacente en ciertas intuiciones modales, es importante mencionar que recientemente Tahko $(2017,30)$ ha desarrollado lo que denomina una propuesta híbrida de epistemología modal, empirically-informed modal rationalism. Siendo uno de sus objetivos principales relevar los aspectos que fortalecen un avance epistémico que se nutra de ambas aristas, a priori-a posteriori, una arista particularmente interesante lo constituye el rol que cumplen las intuiciones y lo concebible (conceivable) en esta propuesta. El punto central en discusión es si la epistemología modal, entendida como un campo de análisis principalmente apriorista y contrafáctico, tiene alguna vía de justificación epistémica válida. No se trata, como pudiese parecer a primera vista, de descartar que un sujeto $\mathrm{S}$ comprenda enunciados como "Posiblemente lloverá mañana", y conozca a partir de ellos. Se trata más bien de si el contenido de proposiciones modales dependen en última instancia de información empírica ${ }^{8}$ (de aquí la postura paralela denominada empirismo modal) o si los enunciados modales contribuyen con alguna información a la que no se puede acceder solo mediante dicha contrastación empírica. Ello quiere decir que una propuesta de racionalismo modal empíricamente informada no renuncia a un estatuto específico de información por ejemplo conceptual que nutra lo que un sujeto $\mathrm{S}$ puede conocer vía a priori, sino que exige como criterio de validación epistémica que dicho ítem considere ya como justificador (warranted) ya como derrotador (defeater) de la información empírica disponible. Un supuesto cuestionable del debate en torno al rol evidencial de cierta intuición es si la evidencia puede ser neutral, no sujeta a ciertos objetivos teóricos o posiciones (posits) filosóficas específicas. Si la evidencia no es neutral, no puede entenderse la misma como una potencial premisa que permita llegar a un consenso metodológico como conclusión esperada. Para que un cierto ítem intuicional se comprenda como evidencia de $\mathrm{p}$ debe, acorde con Goldman, tener un proceso causal (causal process) que permita determinar la probabilidad de que cuando $S$ intuye $\mathrm{p}$, sea probable que p es verdadero ${ }^{9}$. ¿En qué antecedentes causales reside, entonces, la confiabilidad de una intuición? El contraste respecto de qué constituye evidencia correcta o incorrecta es, desde este punto de vista, una cuestión de segundo orden, en tanto evalúa si en este caso un estado mental cumple ciertas condiciones para que se comprenda como un conductor de verdad confiable, más allá de que aquello que se entiende como verdadero lo es independiente de este conductor específico: independiente de si p es intuido o no. De allí la dispensabilidad intuicional, defendida en Miranda (2018).

\section{FALIBILIDAD INTUICIONAL, CALIBRACIÓN Y EVALUACIÓN ALÉTICA}

Deutsch (2015) recientemente ha considerado la posibilidad de una distorsión pragmática (pragmatic distortion) en la elaboración de los experimentos que cuestionan el rol evidencial de las intuiciones en el ámbito filosófico. Dicha noción establece que es una posibilidad que el significado que un sujeto $\mathrm{S}$ le otorga a los casos sometidos a evaluación pueden ser distintos que los pretendidos por el experimento en cuanto tal (su objetividad semántica, o que el significado no varíe entre los sujetos encuestados). Goldman plantea que someter a evaluación ese tipo de intuiciones puede ser la explicación de la variabilidad intuicional. No tendría los mismos resultados, eventualmente, si el criterio de clasificación corresponde a aquellos cuyo estatuto 
no depende fuertemente del estado intencional del sujeto evaluado, los free floating clasificadores: In saying that the specified policy is unjust, he means to classify it relative to a free-floating classifier, not relative to a person-specific or community-specific classifier (Goldman 2013, 22). Las condiciones de satisfacción (satisfaction condition) varían dependiendo de si lo que se pretende evaluar es si un sujeto $\mathrm{S}$ tiene determinado concepto, o si alguna instancia de dicho concepto se adecua a como ese sujeto $S$ entiende ese concepto: presuponer que el contenido del concepto coincide con la comprensión que el sujeto $S$ realiza redunda en que el análisis experimental solo confirma que dicho sujeto $\mathrm{S}$ posee el concepto evaluado, y detecta cuando cierta instancia del mismo es correcta o incorrecta (si por ejemplo la noción de esclavitud es aplicable como un caso de justicia, o no). El nexo evidencial con el valor de verdad de cierta proposición p no reside por tanto en que cierto sujeto $S$ intuya que $\mathrm{p}$, sino en que

ii) Intuye que $\mathrm{p}$ porque $\mathrm{p}$ es verdadero.

Aquellos casos en los que un sujeto $S$ intuye p y $\mathrm{p}$ es falso corresponde al potencial error que un acceso epistémico falible supone. Por supuesto, aquí adquiere relevancia el dato estadístico de que cierta intuición tenga un mayor porcentaje de aciertos que de errores: no sería viable sostener la confiabilidad si respecto de cierto ítem de creencia o conocimiento un sujeto $S$ yerra la mayoría de las veces. Ello redundaría en la exigencia desarrollada por Machery como conclusión de su argumento de la no confiabilidad, cuyo esquema es el siguiente:

"1. Judgments that lack epistemic property $P$ are severely deficient from an epistemic point of view.

2. Judgments elicited by most of the philosophical cases that have been examined by experimental philosophers lack $P$.

3. If the judgments elicited by most of the philosophical cases that have been examined by experimental philosophers lack $P$, then the judgments elicited by most philosophical cases plausibly lack $P$.

4. We ought to refrain from making a judgment of a particular kind K (i.e., we ought to suspend judgment of kind $\mathrm{K}$ ) when most judgments of this kind are plausibly severely deficient from an epistemic point of view, except when this judgment is known to be an exception.
5. Hence, except when a philosophical case is known to elicit a judgment possessing $P$, philosophers ought to suspend judgment about the situations described by philosophical cases" (Machery 2017, 92).

Este argumento asume que una intuición carece de una característica $P$, que desde el análisis de Machery corresponde a la confiabilidad, evaluada desde la ausencia de calibración (Cummins 1998), entendida esta como: ... a source of evidence is calibrated if and only if it has been compared to, and made to agree with, another source of evidence known to be reliable (Machery 2017, 92).

$\mathrm{Si}$, por ejemplo, se exigiese que una intuición fuese sometida a evaluación empírica, de modo tal que potenciales errores de juicio fuesen sometidos y comparados con la información a posteriori disponible, este sería un modo de evitar el problema de calibración. Evitaría, del mismo modo, que se comprendiese como carente de la propiedad epistémica denominada hopefulness: a source of evidence is hopeful if and only if one has the capacity to detect misleading outputs (Ibíd.)

Machery $(2017,96)$ comprende la confiabilidad como una propiedad disposicional (dispositional property) aplicable tanto a procesos psicológicos (psychological process) como inferenciales (inferential process), sin precisar la distinción entre ambos, ni por qué esta confiabilidad se reduciría a evaluar estos procesos. Tampoco considera en detalle los alcances epistémicos de comprender como disposicional y como propiedad la confiabilidad, con los alcances modales que esta disposicionalidad tiene: ¿Es una propiedad que superviene a esos procesos evaluados, es intrínseca a los mismos o surge de una relación sui generis? En efecto, este punto se extiende a las propiedades de hopefulness y calibration, que cumplen desde la perspectiva macheriana un rol meramente instrumental, no dejando claro si son propiedades de las creencias, o solamente aplicables a creencias de modo pragmático o instrumental. Este es una de las aristas centrales del argumento de no confiabilidad, se enmarca en el requerimiento que exige de los juicios filosóficos precisamente tener estas características, a la que Machery agrega la confiabilidad (reliability), explicitando que estas propiedades no tienen un valor intrínseco, de allí su instrumentalidad: 
"Requirement 1: Judgments lacking this property are severely deficient from an epistemic point of view. It is not as plausible to hold that a judgment is severely deficient from an epistemic point of view because it is the product of a hopeless or a non-calibrated process. No doubt, hopefulness and calibration are valuable epistemic properties, but their value is instrumental. They are valuable because they endow belief forming processes with various desirable epistemic properties, including reliability... Since hopefulness and calibration are not intrinsically valuable, when we assess judgments epistemically, it is natural to concentrate on what hopefulness and calibration are for, including reliability" (Machery 2017, 100).

No obstante esta observación, es importante destacar que Machery restringe la confiabilidad como propiedad disposicional a un ambiente específico (environment $E^{10}$ ), si bien esta esquematización se acerca bastante a la empleada en los casos de la filosofía de sillón criticada, sobre todo en vistas a su abstracción y generalización, precisamente un desiderátum inferencial macheriano explícito en la premisa 3 (y de algún modo extendible a la premisa 4). Más importante aún, Machery $(2017,102)$ reconoce no solo la posibilidad sino la existencia de casos científicos en los que se puede cumplir el criterio de confiabilidad sin los dos restantes, lo que indica que no serían condición necesaria para el argumento. Es crucial para el argumento de la no confiabilidad mantener esta arista inferencial, y así evitar la contraargumentación denominada burden-shifting strategy (Williamson, 2011, 2016), cuya principal crítica es la extensión de ciertas conclusiones experimentales a casos no analizados, corriendo por tanto el riesgo de sobregeneralizar (overgeneralize) los resultados $\mathrm{y}$, peor para los objetivos metodológicos, derivar a en la universalización (falible, sujeta a permanente evaluación) de sus conclusiones, punto central de crítica a la metodología tradicional apriorista. Una consecuencia de esto es que no debiesen ser el análisis de los juicios filosóficos una excepción a este criterio de sobregeneralización, menos aún si no hay un compromiso específico con qué determina un juicio como miembro de este tipo de juicios, arista central de la premisa 5. La defensa del paso inductivo que permite la generalización (pues desde una perspectiva experimental negativa no se comprende como sobregeneralización) es el que los experimentos desarrollados consideran los casos típicos (typical cases):

"A philosophical case is typical if and only
if it possesses many of the properties many
philosophical cases possess. For this reason,
everything else being equal, typicality is
a good cue for induction, and atypicality a
good cue that induction should be avoided"
(Machery 2017, 109. Énfasis de quien escribe).

Este paso parece suponer que la cercanía entre casos analizados permite extender las conclusiones más allá de los mismos, pero si se consideran los casos análogos perceptuales, es patente que ostenta la misma similitud: serían casos perceptuales típicos. Más allá de esto, el problema de la inducción es hasta cierto punto ortogonal a la discusión del argumento de la no confiabilidad, pues se supone que incluso si no fuese extendible a casos no analizados, el argumento aplicaría a los experimentos sí realizados. Pero, siendo precisamente ese el supuesto rechazado (que los experimentos efectivamente hayan conseguido ese objetivo (Deutsch 2015)), el paso inductivo es relevado como una arista central del análisis, pues las conclusiones ya no se restringirían al caso a caso. En efecto, la apelación al criterio ceteris paribus no hace más que complicar la posición experimental negativa, pues ese ceteris paribus debiese considerar el ambiente (environment $E$ ), aspecto que difícilmente se puede sostener cumple con esa condición que permite dar el paso inductivo. En otras palabras, el problema del paso inductivo parece tocar el problema de la replicación (punto que no se desarrollará en este escrito). Una arista subyacente a esta apelación a la tipicalidad (typicality) de los casos analizados es que da luces de que el

iii) proyecto experimental negativo cuestiona los casos filosóficos usando estos mismos casos en sus evaluaciones empíricas, lo que presenta una tensión metodológica y plantea la posibilidad de que el proyecto sea fundamentalmente reaccionario.

Sumado a lo anterior, la tipicalidad aplicada a casos filosóficos corresponde a su inusualidad: lo típico de estos casos es que no son usuales, y de ello se derivaría su no confiabilidad: ...philosophical cases are often unusual, and we should expect the judgments they elicit to be unreliable (Machery 2017, 115). 
Machery $(2017,117)$ deja claro que estas nociones no son modales, si bien no discute extensamente el carácter modal del criterio central de su argumento: la confiabilidad. Parece concluir esto del descarte de coextensividad típico-usual/atípico-inusual: Actual cases can be atypical, and merely possible cases can be typical. Being atypical is not simply being unusual (Ibíd.) A su vez, determina el carácter de usual desde su frecuencia temporal (occurs infrequently), lo que pone en cuestión en qué sentido una situación contrafáctica puede ser prima facie no usual en este sentido, considerando que no es posible evaluar su frecuencia temporal. Este es otro punto que no es desarrollado en detalle por la propuesta macheriana, si bien se puede especular que comprender los casos filosóficos como inusuales está fundado en esta comprensión de que dichos casos separan características que en situaciones actuales (no contrafácticas) suelen estar unidas, y de ello colige Machery no confiabilidad. La no confiabilidad no permite establecer a qué tipo pertenece cierta situación u objeto, como el ejemplo de si un ascensor (lift) cuenta o no como un vehículo, lo que es desarrollado por Goldman per se desde un análisis conceptual que no descarta las vías a priori como válidas; no se trata de que ese análisis sea suficiente, pero sí parece necesario, y la sola información empírica no conduce per se a una clasificación correcta confiable. La relevancia de una clasificación correcta reside en que sin la misma no es factible restringir la no confiabilidad solo a casos filosóficos, evitando así el riesgo de un escepticismo global judicativo.

Si el argumento de la no confiabilidad intuicional alcanza su conclusión respecto de las intuiciones a partir de encuestas que no evalúan directamente lo analizado en la sección anterior (el carácter evidencial de cierto estado mental) sino que más bien la variabilidad entre sujetos y culturas como un modo de cuestionar la objetividad de los insumos que esas intuiciones entregarían, lo que se está cuestionando no es el contenido del estado mental, sino el estado mental per se como conductor de verdad. El problema de esta estrategia es que supone que el contenido de lo intuido no es evaluable aléticamente. Probablemente es por ello que al análisis experimental negativo no le interesa someter a crítica las intuiciones no expertas (lay intuitions) en otras áreas del conocimiento cuya objetividad o validez no intenta ser puesta en tela de juicio. El ataque a los casos específicamente filosóficos tiene por objetivo descartar que aquello que no pueda ser verificable sea objeto de conocimiento o de creencia. Si ello fuese extendido a otras ramas del saber, llamaría la atención que tenga tal impacto en el ámbito filosófico: en otras áreas simplemente no sería relevante las creencias no expertas en vista a determinar la confiabilidad de lo que los expertos consideran verdadero. Esta ausencia de generalidad respecto del análisis intuicional solo pone en cuestión la metodología que cuestiona en primer lugar el rol evidencial de las intuiciones, independiente de si las mismas cumplen finalmente o no ese rol. Es, además, un doble estándar epistémico:

iv) confiar en las intuiciones no expertas como una vía de defeater del uso de las intuiciones en al ámbito epistémico.

$\mathrm{Al}$ evaluar la potencial relevancia de las intuiciones y los antecedentes de su variabilidad empírica entre distintos individuos y distintos sujetos, una pregunta que ha quedado relegada en la discusión actual es qué rol específico cumplen esos datos empíricos relevados por la filosofía experimental negativa como evidencia de un error metodológico en filosofía. Por ello, la interpretación desde una posición racionalista naturalista (Miranda, 2018b), que admite una relevancia fenomenológica de la contrastación empírica de las intuiciones racionales, evidencia de que es el caso que cierto sujeto $S$ o grupo de individuos afirma o cree p. Sin embargo, ello no toca el punto filosófico relevante de fondo ¿Por qué $S$ intuye y cree $\mathrm{p}$ ? ¿Es contrastable empíricamente lo que $S$ intuye? Si no lo es ¿Es un sinsentido que $S$ intuya p? A esta situación metodológica compleja propongo denominarla

v) la paradoja experimental: desconfiar del sentido común usando el sentido común.

El dato que la filosofía experimental confirma es que $\mathrm{s}$ intuye $\mathrm{p}$, el dato empírico de que $\mathrm{s}$ intuye p. En otras palabras, siguiendo a Goldman $(2013,17)$

vi) variabilidad intuicional no implica directamente no confiabilidad.

La conclusión de que ciertos factores aléticamente irrelevantes (Alexander y Weinberg 2007, 61) influyen en lo que ciertos sujetos intuyen en ciertos experimentos controlados, da cuenta de que 
es importante preguntar qué determina la irrelevancia de esos factores (orden de presentación, estrato sociocultural, raza) si no es el contenido de lo que es intuido; es sostener que los contextos de presentación de un caso intervienen o modifican el contenido de los enunciados que los sujetos deben leer e interpretar en orden a afirmar qué alternativa les parece más intuitiva, o más correcta, entendiendo lo intuitivo como lo correcto. Machery $(2017,104)$ denomina estos factores normatively inappropriate influence, en su ejemplo el que el color de la sala en la que se realice un experimento (hipotético en este caso) relacionado con el cambio climático, influya en los resultados. Lo que es interesante, es que el autor descarta que esto sea en efecto un factor que debiese (considerando la arista normativa) afectar estos juicios, y que el foco del argumento de la no confiabilidad es que independiente de ello (que no deban influir), el hecho de que estos factores influyan en el juicio de un sujeto $S$ resta confiabilidad (reliability) al juicio del mismo:

\begin{abstract}
"The color of the room should not influence one's opinion on global warming, but this plays no role in the argument here. What only matters here is whether its influence undermines the reliability of judgment" (Ibíd.)
\end{abstract}

Goldman observa que estos factores irrelevantes rompen el nexo causal que otorga confiabilidad evidencial en primer lugar a ciertas intuiciones:

vii) si las intuiciones varían con motivo de estas modificaciones irrelevantes, la pregunta que subyace es si se asume una posición universalista respecto de que ante dos intuiciones opuestas a y b, solo una es correcta, o verdadera.

Si el supuesto descarta que puedan ser ambas erradas o ambas correctas (por su ulterior inconsistencia de creencias), parece seguirse desde la postura experimental una exigencia de ausencia de variabilidad intuicional, una postura universalista:

viii) el objetivo último sería encontrar un consenso entre intuiciones como evidencia de que son un acceso confiable a cierta información, siendo esta por tanto objetivamente verdadera.

Es extraño que una postura que tiene entre su foco cuestionar el anhelo de universalidad de ciertas intuiciones filosóficas, establezca como supuesto base de sus experimentaciones la condición de que ix) si no hay universalidad, no hay acceso confiable al conocimiento, en este caso al estatuto de cierta creencia.

Observa Goldman que lo que se requiere desde la postura experimental es evidencia de segundo orden que muestre por qué el estatus evidencial de las intuiciones en una perspectiva de primer orden, no es un acceso epistémico confiable. De este modo, la postura escéptica restriccionista respecto de las intuiciones (Alexander y Weinberg 2007) cuestiona el rol de las mismas como evidencia del valor de verdad de ciertas afirmaciones.

Hay, no obstante, una alternativa que si bien rescata un rol evidencial indirecto, no compromete el análisis filosófico con una toma de posición respecto de si una proposición p es intuitivamente verdadera o falsa. Denomino a este rol propedéutico o ejemplificador contrafáctico, y su principal característica es permitir que un sujeto $S$ evalúe los distintos escenarios contrafácticos que los casos hipotéticos plantean como problemática filosófica. Que $S$ intuya que $p$ es verdadero o falso constituye un paso ulterior a dicha comprensión prima facie, que en este sentido puede ser entendida como una disposición a creer (disposition to believe) o no creer que es el caso que p. Es correcto que la elaboración de un experimento mental puede dirigir, tal vez de modo sesgado, a cierta conclusión específica. Pero

$\mathrm{x}$ ) el proceso de evaluación que un sujeto $\mathrm{S}$ realiza del caso en cuestión no le compromete prima facie con aceptar esa pretendida conclusión.

Esto se alinea con el falibilismo apriorista propuesto por Casullo (2003), y que es relevado por el análisis evidencial de segundo orden, de carácter empírico, analizado por Goldman (2013). En realidad, es esta intuición de base la que guía la propuesta experimental contemporánea, pese a que dicha propuesta no acepte la ubicuidad del rol intuicional incluso en evaluaciones empíricas: el planteamiento de la hipótesis inicial de los diversos experimentos pretenden precisamente evaluar los diversos escenarios posibles y, de este modo, hay implícito un análisis contrafáctico previo a la evaluación de los resultados. Esto rescata el desiderátum central de la propuesta confiabilista analizada por Goldman: 
"Part of the task of deciding whether classification intuitions have evidential value for philosophy is the task of deciding which hypotheses or conclusions (of philosophical interest) they might be evidence for" (Goldman 2013, 19).

\section{NEUTRALIDAD METAFÍSICA Y MODESTIA FILOSÓFICA: ALGUNAS CONSECUENCIAS DE UNA PROPUESTA INTUICIONAL RACIONALISTA MODERADA}

Si los casos filosóficos no son confiables ¿De dónde proviene la relevancia de discutirlos, y no descartarlos prima facie como el caso análogo del flogisto? El punto metodológico de fondo es relevante $i$ Son las aristas analizadas (etnicidad, estrato socioeconómico, nivel de experticia, modo de presentación, inter alia) variables dependientes o independientes de la propuesta experimental? Si son dependientes, se comprendería la apelación al ceteris paribus como criterio que permite la inducción desde casos usuales. Pero ¿son variables dependientes? Parece complejo sostener que lo sean, son tratadas más bien como antecedentes del experimento que eventualmente pueden explicar la diferencia o diversidad intuicional de los resultados, las características que perturban (disturbing characteristics. Machery 2017, 111) los resultados y que inciden en la variabilidad intuicional, y su aludida falta de confiabilidad. Es paradójico, por tanto, que se pretenda mediante el criterio ceteris paribus realizar aquello que se critica a los casos filosóficos:

xi) abstraer aspectos que desde una perspectiva etiológica incidirían en los juicios intuitivos (o snap judgment, como lo denomina Machery) de un grupo de individuos.

Ulteriormente, Machery aplica implícitamente el criterio de ceteris paribus aplicado a la ley de gravedad, ejemplo interesante pues es usado para establecer una diferencia entre

xii) situaciones hipotéticas filosóficas, no confiables; y situaciones hipotéticas fundadas en alguna ley natural (lo que Reutlinger et alia denominan ceteris paribus laws), confiables.

Afirma Machery:

\begin{abstract}
"One could speculate that the hypothetical nature of the examined philosophical cases explains...why the elicited judgments are unreliable, but there is in fact little reason to believe that judgments about hypothetical situations are in general unreliable: Simply consider the judgment that this book would fall instead of going up if you released your grip on it (Machery 2017, 113).
\end{abstract}

Como indica Reutlinger et alia (2019): Since many philosophers have argued that ceteris paribus laws lack empirically testable content, this problem constitutes a major challenge to a theory of ceteris paribus laws". Por tanto, no es completamente indiscutible el uso de un supuesto como este, en orden a validar el paso inductivo del argumento de la no confiabilidad. Además, no hay solo un intento de generalización, sino de universalización (que se aplique a todos los casos típicos). Ulteriormente, Machery va un paso más allá y sostiene que los casos filosóficos violan ciertas leyes naturales, obviando que su carácter contrafactual permite precisamente esto, siendo un presupuesto de dichas situaciones contrafácticas la posibilidad (o al menos la concebibilidad, Chalmers 1996) de que ciertas leyes naturales sean distintas, en el caso hipotético:

\begin{abstract}
"Many of the non-actual situations that are most telling to assess such philosophical views are unusual either because they violate the laws of nature (e.g., thought experiments involving zombies, water on Twin Earth, split brains, etc.) or because they involve situations that are extremely different from what happens in the actual world" (Machery 2017, 116).
\end{abstract}

Es por supuesto discutible este supuesto modal que fundamenta casos concebibles (ver Miranda 2017), pero descartar esta posibilidad no es precisamente un caso de modestia filosófica, sino por el contrario afirmar la necesidad fuerte de las leyes naturales: es una postura metafísica específica. Esta arista muestra que el supuesto metafísico naturalista, y el no suspender el juicio respecto de este compromiso metaepistémico, es central en la discusión. No es un problema per se este compromiso, lo es el no hacerse cargo del mismo y pretender una cierta neutralidad metafísica, lo que se ha evidenciado no es el caso desde el análisis desarrollado previamente, considerando el compromiso de la filosofía experimental con un 
paradigma empirista (fuerte o débil, dependiendo de si se considera la perspectiva positiva y negativa de esta corriente filosófica heredera del naturalismo $)^{11}$. Conjuntamente, la ausencia de neutralidad es uno de los focos de la crítica macheriana al método de $\operatorname{casos}^{12}$. Ante el análisis de conceptos filosóficamente relevantes (conocer, libertad, causalidad, responsabilidad, etc.), es admisible destacar el posible carácter polisémico de algunos, como conocer, de tal modo que la experimentación intercultural pudiese reflejar no intuiciones distintas respecto de un mismo concepto, sino de conceptos diferentes:

\begin{abstract}
"Suppose that a person-specific content is our favored type of classifier, and that different people associate different contents with a term of philosophical interest. When invited to decide whether "knows" applies to a specified case, one respondent uses his concept of knowing, K1, while another uses her concept of knowing, K2. If the first makes a positive classification judgment and the second makes a negative one, their verbal responses suggest contradictory intuitions, so one of them must be in error. But if their judgments "answer" different questions, it does not follow that either is in error. Each might answer correctly in terms of his/her own concept, or conception, of knowing. Thus, choosing person-specific contents as the classifier would lower the error rate. This would result in higher reliability scores for intuitions and possibly avert intuition skepticism" (Goldman 2013, 22).
\end{abstract}

Si este es el caso, siguiendo a Goldman, la insensibilidad (insensitivity) a esta pluralidad de significados (tanto semántico como pragmático) pone en riesgo la evaluación de las intuiciones que diversos sujetos tienen, dependiendo ellas eventualmente de distintos sentidos (o significados, considerando lo mencionado previamente). Así Goldman (2013, 27) enuncia tres potenciales alternativas respecto de qué entiende un sujeto $\mathrm{S}$ por conocer:

i) completely confident of it (estrictamente en este caso el autor se refiere al gerundio knowing)

ii) to believe something truly

iii) justified-true-belief-plus (también referido al gerundio knowing).

Sostiene que, debido a que la evaluación de competencia conceptual asume la comprensión que
$\mathrm{S}$ tiene, no es una alternativa que $\mathrm{S}$ cometa un error al clasificar alguna instancia como caso del concepto (Ludwig 2007), pues es un presupuesto. Más importante, la probabilidad de que el rol evidencial sea correcto aumenta si en lugar de considerar casos individuales, se toman en consideración las intuiciones de un conjunto de individuos, la perspectiva social intuicional que sostiene Goldman apoyado en el teorema del jurado Cordorcet (Cordorcet Jury Theorem), el que permite sostener aumenta la probabilidad de que (en este caso) ciertas intuiciones sean correctas si cumplen tres condiciones: (1) there are two options, (2) each group member has a competence greater than $1 / 2$, and (3) the members'beliefs are conditionally independent of one another (Goldman 2013, 32).

No obstante, este teorema solo aumenta la probabilidad de que una intuición sea correcta, solo presuponiendo que una de las dos opciones enunciadas en 1 es la mejor opción. Ocurre, en los casos de análisis intuicional, que es precisamente eso lo que debe ser determinado, y no es por tanto un presupuesto de la evaluación que cierta intuición A es correcta en comparación con su negación, no - A. Ocurre, por tanto, que el supuesto en juego es que la intuición correcta es la intuición de la mayoría, ella sería evidencia central de su confiabilidad. La variabilidad intuicional entre culturas, de este modo

xiii) no redundaría en una pérdida de confiabilidad de este acceso epistémico, sino que permitiría observar la importancia de no comprender de modo solipsista los juicios intuitivos, sino en un contexto sociocultural específico (lo que Machery denomina environment $E$ ).

A su vez, esto le permite evitar el problema de la generalización (generality problem, Machery 2017,98 ) el que exigiría en primer lugar explicar cómo ciertos juicios permiten justificar o conocer por medio del criterio de confiabilidad. Observa Machery que este problema de una postura confiabilista puede ser evitado si se restringe al análisis de un tipo de juicios en particular, sin abordar el problema de si todo juicio requiere un proceso confiable para estar justificado o ser un acceso legítimo a cierto conocimiento. Esto es un problema, pues supone que puede abordarse cierto juicio $\mathrm{J}$ de un tipo $\mathrm{T}$ (philosophical cases), sin tener una tipología judicativa general. ¿Cómo puede determinarse entonces que este juicio $\mathrm{J}$ no es de un tipo distinto 
a T? La respuesta de Machery $(2017,99)$ es circular: Types are given to us in the formulation of our problem... We single out a given type because we have no information about partitions of this type resulting in subtypes with different reliabilities.

Los alcances escépticos de esta argumentación descansan en que tópicos centrales del ámbito filosófico (conocimiento, justificación, responsabilidad moral, inter alia) no sean considerados como propiedades naturales (Machery 2017, 103), cuya principal implicación sería que no existen independiente de los estados intencionales de un sujeto $\mathrm{S}$. Que una posición escéptica se apoye en esta posición metafísica específica solo muestra la tensión entre

xiv) una postura epistémica que intenta cuestionar los alcances del análisis filosófico tradicional, y a la vez establecer criterios que surgen al menos parcialmente desde análisis conceptuales propios de esa tradición criticada.

Es probablemente otra consecuencia indeseada de la paradoja experimental enunciada previamente, y tal vez por ello la versión del escepticismo macheriano es débil, mera suspensión de juicio: un caso de agnosticismo que entra en tensión con el descarte previo de análisis intuicionistas y aprioristas en general, pues la acusación de dogmatismo y estrechez mental (parochialism, Machery 2017, 10) proviene precisamente de confiar en fuentes y accesos epistémicos que no solo serían falibles, sino tendientes al error más que a lo correcto. En efecto, el paso siguiente es cuestionar que estos juicios tengan siquiera un objeto:

“...assessing judgments about knowledge, justification, or moral permissibility for their reliability supposes that there is something -whether natural or not- the relevant judgments are about. It makes no sense to assess judgments about witches, ghosts, or phlogiston for their reliability. Similarly, if responsibility is a fiction, it makes no sense to assess the reliability of judgments about responsibility" (Machery 2017, 103).

Es sin duda una postura metafísica fuerte y no neutral sostener que la responsabilidad sea una ficción, siendo estricto, la posibilidad de que lo sea (es un condicional pues inicia con if). Considerando lo anterior, el escepticismo macheriano pretende ser solo de carácter epistémico, intentando evitar abordar estos puntos en discordia respecto de la arista metafísica, y también la semántica ${ }^{13}$. Sin embargo, ello es pretender definir si cierto juicio tiene algún hacedor de verdad (truth maker) que permite que un cierto enunciado sea comprendido como un portador de verdad (truth bearer), sin pronunciarse primero respecto de qué sería aquello verdadero o falso. Y los casos de obviedad no confiabilista como los ejemplos del flogisto (polémico ejemplo considerando sus antecedentes); las brujas o los fantasmas, no hace más que relevar una tensa posición entre el desiderátum de restringirse a lo epistémico, mediante ejemplos de obviedad no confiabilista que se apoyan fuertemente en el estatuto metafísico de su pretendido objeto (o la ausencia de este).

Entonces, la pregunta metodológica que debe plantearse es ¿Por qué juicios irrelevantes e injustificados requerirían de algún defeater específico? Si es tan obvio que son conducentes a error en lugar que a lo verdadero, como los ejemplos análogos sugieren ¿De dónde surge siquiera la necesidad de establecer epistémicamente tanto undercutting defeaters como rebutting defeaters? Actualmente, parece ser que la respuesta a esto es que no es un enfoque primariamente epistémico el que dirige esta discusión, sino uno de carácter de compromisos metafísicos subyacentes, que son en primer lugar la oposición presente entre naturalismo y racionalismo, y la filosofía experimental como la vertiente más reciente del primero. Dicho de modo más claro si se puede:

xv) No hay una suerte de neutralidad metafísica en esta discusión, y pretender que así sea solo oscurece los presupuestos teóricos en juego.

Es preferible, por tanto, determinar una vía colaborativa, racionalista naturalista, que permita descartar intuiciones que conducen a error (como las ilusiones modales o su análogo de las ilusiones perceptuales) sin que ello implique inferencialmente (de acuerdo con el argumento de la no confiabilidad) cometer el error de generalización apresurada. Sea como sea, el argumento de la no confiabilidad permite visibilizar estas aristas metafilosóficas en juego, sin estas tampoco será posible (si es posible) superar el principal foco de crítica a la filosofía de sillón: la ausencia de progreso filosófico y el permanente desacuerdo (disagreement) entre diversas posturas. La contrastación empírica sin duda cumple un rol 
relevante en este desafío metodológico de la reflexión filosófica, pero no agota ni reduce la misma. Al sostener que la no confiabilidad de los casos filosóficos es fundamental, Machery $(2017,116)$ parece cometer el error que atribuye a la filosofía tradicional: inmodestia filosófica. Tal vez peor, sostener el argumento de la no confiabilidad y su consecuencia de suspensión de juicio en la reflexión filosófica, impediría del mismo modo sostener la confiabilidad del argumento mismo, al extraer los datos empíricos que lo fundamentan desde la variabilidad intuicional en diferentes culturas. No se trata de una reductio ad absurdum, pero sí releva ciertas aristas problemáticas de la dialéctica argumentativa experimental, y de las consecuencias metodológicas que espera extraer de los resultados empíricos de sus experimentos. Los argumentos desde la filosofía experimental no se basan ni dependen de intuiciones, pero sí usan estas (tanto las expertas como las no expertas o lay intuitions) como un insumo relevante de los experimentos empíricos que evalúan las respuestas de diversos sujetos cognoscentes ante casos hipotéticos similares a los experimentos mentales ${ }^{14}$. Al evaluar la potencial relevancia de las intuiciones y los antecedentes de su variabilidad empírica entre distintos individuos y distintos sujetos, una pregunta que ha quedado relegada en la discusión actual es qué rol específico cumplen esos datos empíricos relevados por la filosofía experimental negativa como evidencia de un error metodológico en filosofía. En este escrito se ha intentado defender una posición racionalista naturalista que admite una relevancia fenomenológica de la contrastación empírica de las intuiciones racionales: evidencia de que es el caso que cierto sujeto $S$ o grupo de individuos afirma o cree p. Sin embargo, este análisis experimental negativo no toca el punto filosófico relevante de fondo ¿Por qué $\mathrm{S}$ intuye y cree $\mathrm{p}$ ? ¿Es contrastable empíricamente lo que $\mathrm{S}$ intuye? Si no lo es ¿Es un sinsentido que $\mathrm{S}$ intuya p? La paradoja experimental reside en desconfiar del sentido común, usando el sentido común. Por tanto, una consecuencia intuicional positiva es que la filosofía experimental negativa confirma que $S$ cree p, el dato empírico de que s cree p. En otro escrito discutiré los supuestos y alcances de estos resultados.
Para terminar, quisiera citar un ejemplo reciente de cómo ciertas intuiciones a priori pueden guiar una discusión fructífera, independiente de si no se logra el criterio de acuerdo filosófico enunciado. Corresponde al caso de la justificación respecto del cese de la esclavitud, que si bien se fortalece evidentemente con información empírica esbozada en esa cita, la argumentación respecto de las intuiciones morales no se reduce a ella, lo que muestra lo que se ha intentado discutir en este escrito: que las intuiciones sí constituyen un aporte epistémico, y que suspender el juicio o tomar una postura escéptica respecto de las mismas pudiese no ser la mejor opción:

\footnotetext{
"A...objection is that many human practices... violate moral intuitions that we doubt that our moral intuitions can have much force...let me take up one or two examples...Slavery has been practiced by virtually every culture in the history of the world, and it is still practiced today in some remote places... How is that people can engage in slavery and still be said to possess moral intuitions?...the argument about slavery is an argument about the effort to generalize the principles upon which one's moral intuitions rest. Generalizing moral principles is a long and anguishing process in human development...for example, how do you justify black slavery? The only coherent principle is to say that blacks are inferior to whites. You could make some practical arguments, but the practical arguments would admit of the possibility of exempting some blacks from slavery, because they satisfy the practical concerns that would otherwise justify slavery. But if we say that all blacks are inferior to all whites we are making a mistake. Abraham Lincoln put it...there is no principle that can justify black slavery that will not justify the slavery of some whites... Otherwise it would be the case that the most depraved white criminal was superior to the most gifted black intellectual, and obviously that cannot be true. If you cannot justify slavery by universalizing the principle of slavery, then slavery... will be destroyed by people who point out this contradiction" (Wilson 2000, 32).
} 


\section{BIBLIOGRAFÍA}

Alexander, J. and Weinberg, J. M. (2007). Analytic epistemology and experimental philosophy. Philosophy Compass 2 (1): 56-80.

Bealer, G. (1998). Intuition and the autonomy of philosophy. En DePaul and Ramsey (eds), Rethinking intuition: The psychology of intuition and its role in philosophical inquiry. Rowman and Littlefield.

BonJour, L. (1980). Externalist Theories of Empirical Knowledge. Midwest Studies in Philosophy, 5: 53-74.

Casullo, A. (2003). A priori justification. Oxford University Press.

Chalmers, D. J. (1996). The conscious mind. Oxford University Press.

Chalmers, D. J. and Jackson, F. (2001). Conceptual analysis and reductive explanation. Philosophical Review 110 (3): 315-60.

Cummins, R. (1998). Reflection on reflective equilibirum. En M. R. DePaul and W. Ramsey (Eds.), Rethinking intuition: The psychology of intuition and its role in philosophical inquiry (pp. 113-28). Rowman and Littlefield Publishers.

Gettier, E. (1963). Is justified true belief knowledge? Analysis 23: 121-3.

Goldman, A. I. (1976). Discrimination and perceptual knowledge. The Journal of Philosophy, 73, 771-91.

Goldman, A. I. (2013). Philosophical naturalism and intuitional methodology. En A. C. Thurow (Ed.), The a priori in philosophy (pp. 11-45). Oxford University Press.

Hetherington, S. (2013). Concessive knowledge-attributions: Fallibilism and gradualism. Synthese, 190 (14), 2835-2851. Retrieved October 7, 2020, from http://www.jstor.org/ stable/24021413

Huemer M. (2005). Ethical Intuitionism. Palgrave Macmillan.

Ichikawa, J. (2009). Knowing the intuition and knowing the counterfactual. Philosophical Studies 145 (3): 435-43.

Ichikawa, J. and Jarvis, B. (2009). Thought-experiment intuitions and truth in fiction. Philosophical Studies 142 (2): 221-46.

Ichikawa, J. (2014). Who needs intuitions? Two experimental critiques. En A. R. Rowbottom (Ed.), Intuitions (pp. 232-255). Oxford University Press.

Kelly, T. (2008). Evidence. The Stanford Encyclopedia of Philosophy, E. N. Zalta (ed.), http://plato.stanford.edu/archives/ fall2008/entries/evidence/

Kripke, S. (1980). Naming and necessity. Harvard University Press. Lehrer, K. (1990). Theory of knowledge. Westview Press.
Ludwig, K. (2007). The epistemology of thought experiments: First person versus third person approaches. Midwest Studies in Philosophy 31.

Lycan, W. (1988). Moral facts and moral knowledge. Judgement and justification. Cambridge University Press.

Machery, E. (2009). Doing without concepts. Oxford University Press.

Machery, E. (2015). The illusion of expertise. En E. and Fischer, Experimental Philosophy, Rationalism, and Naturalism: Rethinking Philosophical Method (pp. 188-203). New York : Routledge.

Machery, E. (2017). Philosophy within its proper bounds. Oxford University Press.

Machery, E. (2019), Précis of philosophy within its proper bounds. Philos Phenomenol Res, 98: 221-229, doi:10.1111/phpr.12566

Papineau, D. (2020) Naturalism. The Stanford Encyclopedia of Philosophy. Edward N. Zalta (ed.), https://plato.stanford.edu/ archives/sum2020/entries/naturalism/

Reutlinger, A., Schurz, G. and Hüttelmann, A. (2019) Ceteris paribus laws. The Stanford Encyclopedia of Philosophy, Zalta, E. (ed.) https://plato.stanford.edu/archives/spr2019/entries/ ceteris-paribus/

Tahko, T. E. (2017). Empirically-informed modal rationalism. En B. F. Leon (Ed.), Modal Epistemology After Rationalism. Studies in Epistemology, Logic, Methodology (pp. 29-46). Springer.

Swain, S., Alexander, J., and Weinberg, J. M. (2008). The Instability of Philosophical Intuitions: Running Hot and Cold on Truetemp. Philosophy and Phenomenological Research 76 (1): 138-55.

Weinberg, J. M. (2007). How to challenge intuitions empirically without risking skepticism. Midwest Studies in Philosophy 31 (1): 318-43.

Wilson, J. (2000). Moral Intuitions. Transaction Publishers.

Williamson, T. (2007). The Philosophy of Philosophy. Blackwell.

Williamson, T. (2010). Philosophy vs. imitation psychology. New York Times, August 19, 2010, disponible en http://www.nytimes. com/roomfordebate/2010/08/19/x-phis-new-take-on-old-problems/ philosophyvs-imitation-psychology

Williamson, T. (2011). Philosophical expertise and the burden of proof. Metaphilosophy, 42, 215-29.

Williamson, T. (2016). Philosophical criticisms of experimental philosophy. En J. Sytsma and W. Buckwalter (Eds.), A companion to experimental philosophy (pp. 22-36). New York: John Wiley and Sons. 


\section{NOTAS}

1 La redacción de este escrito se enmarca en el proyecto FONDECYT Iniciación № 11180422 Racionalismo Modal, Naturalismo e Intuiciones, y la Beca de Postdoctorado en el Extranjero BECAS CHILE. Universidad de Pittsburgh - Duquesne University, Pittsburgh - Estados Unidos. Mis sinceros agradecimientos por el respaldo. Agradezco también los comentarios realizados por los/las revisores/as del artículo, que ayudaron a fortalecerlo en diversas aristas.

2 Siguiendo a Papineau (2020), se entiende por naturalismo en un sentido amplio aquella postura filosófica que establece vínculos estrechos con las ciencias. Al mismo tiempo, una posición naturalista puede descartar la relevancia del análisis filosófico en aristas que no se restrinjan al ámbito natural. Pese a esta característica común, Papineau observa que hay un amplio desacuerdo respecto de los alcances del naturalismo en el ámbito filosófico. Afirma: Different contemporary philosophers interpret "naturalism" differently. This disagreement about usage is no accident. For better or worse, "naturalism" is widely viewed as a positive term in philosophical circles -only a minority of philosophers nowadays are happy to announce themselves as "non-naturalists". This inevitably leads to a divergence in understanding the requirements of "naturalism". Those philosophers with relatively weak naturalist commitments are inclined to understand "naturalism" in a unrestrictive way, in order not to disqualify themselves as "naturalists", while those who uphold stronger naturalist doctrines are happy to set the bar for "naturalism" higher. En particular, la tensión se observa en si bajo esta denominación se ubicaría algún tipo de reduccionismo, lo que además depende de la posición teórica que un sujeto $\mathrm{S}$ o un grupo $\mathrm{G}$ favorezca de entrada. La filosofía experimental negativa, foco del análisis de este escrito, se enmarcaría en un tipo de naturalismo fuerte, al descartar otras fuentes de acceso epistémico no naturalistas como válidas, siendo un caso paradigmático el descarte de las intuiciones. Por contraparte, la denominada filosofía experimental positiva restringe el alcance del rol epistémico de las intuiciones, sin descartar su utilidad. Es en vistas a esta segunda alternativa que el presente escrito intenta evitar un escepticismo respecto de las intuiciones. En particular, Machery $(2019,227)$ explicita que su postura naturalista se refleja en lo que denomina análisis conceptual naturalizado, con un enfoque psicológico (psicologizado es el término que usa el autor). Afirma: The psychologized version of conceptual analysis I embrace ("naturalized conceptual analysis") is philosophically significant even if, in contrast to the ambitions of traditional conceptual analysis, it does not dsino eliver truths a priori.

3 Ver Hetherington (2013), en vistas al nexo entre gradualismo epistémico y variabilidad intuicional, en cuyo caso dicha variabilidad correspondería a un caso de gradualidad epistémica entre los entrevistados. Agradezco a un/a revisor/a anónimo/a por compartir la importancia de esta conexión, que espero desarrollar en un futuro escrito.

4 Las afirmaciones numeradas ( i), ii), etc.) en este escrito corresponden a aspectos relevantes de la argumentación desarrollada desde la bibliografía analizada, así como algunos de sus resultados. En particular, se enuncian numeradas en vistas al fortalecimiento de la propuesta del escrito, tal cual es ampliar el alcance de la noción de confiabilidad más allá del rol evidencial epistémico atribuido a las intuiciones, y evitar así el escepticismo modal presupuesto en esa postura naturalista extrema que descarta la validez epistémica de ciertos accesos epistémicos a priori (enfocado en este escrito en las intuiciones). Agradezco a un/a revisor/a anónimo por indicar la pertinencia de esta precisión.

5 Machery $(2019,224)$ menciona principalmente dos características que pueden perturbar ciertos insumos filosóficos: variables demográficas y el orden de presentación de los casos analizados, el carácter inusual de los casos filosóficos, y el que para fines de análisis filosóficos se separen propiedades que se encuentran unidas en situaciones comunes. Sostiene: ...I must explain why we are allowed to generalize from the philosophical cases that have been examined by experimental philosophers to the broader class of philosophical cases. I examine several strategies to justify this induction, but I end up focusing on the typical characteristics of philosophical cases that explain why they elicit unreliable judgments. These characteristics make it likely that the elicited judgments are influenced by demographic variables or by the ways philosophical cases are presented. I call them "the disturbing characteristics" of philosophical cases. Some of these disturbing characteristics are non-accidental: To fulfill their function (i.e., to be brought to bear on the assessment of modal claims), philosophical cases must have these disturbing characteristics. As I say, unreliability is "fundamental". These include the unusual nature of philosophical cases, the fact that philosophical cases pull apart the properties that come together in everyday circumstances (e.g., the properties that instances of knowledge have in everyday circumstances), and the entanglement of superficial and target content. Se observa la relevancia que adquieren estas características, al sostener que son no accidentales y que ello redunda en una falta de confiabilidad intuicional fundamental, o no meramente circunstancial (o accidental).

6 Ver también Huemer $(2005,100)$, quien establece una analogía entre ilusiones visuales (específicamente el caso MüllerLyer, referente a la longitud de dos líneas) y apariencias intelectuales (intelectual appearances). El hilo conductor es que tanto las apariencias perceptuales (este es el término strictu sensu que usa Huemer) como las intelectuales, pueden ser evaluadas de modo tal que si un sujeto S no cree que estas apariencias sean correctas o verdaderas, aún así acepta la apariencia de corrección:

Presumably, it more clearly seems to you that the result of measuring the lines is accurate than that the result of eyeballing them is, so you believe the measurement result (this may have to do with background beliefs you have about the reliability of different procedures-which would themselves be based upon the way other things seem to you). Things can become complicated when many different beliefs and/or appearances are involved, but the basic principle is that we are nlOre inclined to accept what more strongly seems to us to be true.

Esto tiene relación con lo que Huemer denomina phenomenal conservatism (conservadurismo fenoménico), la tendencia a que aquello que parece verdadero (seems true), lo sea.

7 https://www.iep.utm.edu/reliabil/

$8 \quad$ Tahko $(2017,30)$ according to which modal knowledge is purely experiential.

9 El ejemplo es desde la analogía con información y contrastación empírica:

...processes that lead to a state often contribute to its evidential or non-evidential status. Consider our example of the clock that reads 4: 45. Is this reading a reliable indicator of the true time (in the time-zone where the clock is located)? This depends on prior 
events and processes that led to the clock's current reading. For example, when the clock was last set, was it set correctly relative to the time-zone it was in? If so, has it remained in the same timezone until now or has it been moved to a different time-zone? Finally, how well does the clock's time-keeping mechanism work? A defective mechanism, obviously, can make a big difference to the clock's current accuracy. In short, past events and processes that generate the clock's current reading bear on the question of whether the current reading indicates the correct time (in the current time-zone) (Goldman 2013, 15).

10 Ulteriormente, Machery $(2017,107)$ rechaza el contextualismo como vía de solución al escepticismo modal. Ello plantea el problema de qué rol cumple el ambiente (environment $E$ ) en su propuesta, asumiendo que no es determinar el contexto justificatorio. 11 Es importante precisar que este escrito no intenta levantar un argumento contra esta posición metafísica ni los supuestos de la misma, sino más bien precisar los límites de la misma en vistas a la crítica escéptica al intuicionismo. En efecto, Machery (2017) dedica el capítulo 2 a relevar el rol de los hallazgos empíricos en la discusión filosófica, especialmente la ayuda que dichos hallazgos podrían contribuir a una resolución de la falta de consenso filosófico en diversos tópicos, una de las aristas centrales del nexo entre inmodestia filosófica y el denominado método de casos. Afirma Machery (2020, 222): As neutrally described as possible, the method of cases just is the use of cases to assess modal claims. Naturally, cases are used in other ways in philosophy: Sometimes they are used to illustrate the definition of a concept; other times, they are used to formulate a philosophical puzzle. These uses (which I just call "the use of cases") are not problematic, and they are not my concern in Philosophy Within Its Proper Bounds; rather, my concern is with the use of cases to assess modally immodest claims ("the method of cases" properly speaking). Surprisingly perhaps, there is no consensus among philosophers about how to characterize the method of cases: Among other live issues, philosophers do not agree about the nature of the attitude that the reader forms about the situation described by a philosophical case; nor do they agree about the content of this attitude...and there is even a debate about how often (if at all) philosophers use the method of cases. Agradezco a un/a revisor/a anónimo/a por enfatizar la importancia de este punto.

12 El método de casos y una postura empirista no deben ser comprendidos ni como co-extensivos ni como co-dependientes. No obstante, sí existe un compromiso empirista desde una perspectiva experimental, motivo por el que el rechazo al uso del método de casos en la filosofía tradicional sí descansa en una postura empirista (restrictiva) inicial. De allí la relevancia de una propuesta moderada. Así, no se trata de comprometer a una postura experimental con el rechazo sin excepciones de conocimiento a priori, ni de sostener un compromiso indirecto con el método de casos por parte de la propuesta experimental. Se trata, en su lugar, de mostrar las tensiones metodológicas subyacentes, lo que corresponde a una pretensión más modesta que la de derribar vía argumentativa cierta perspectiva filosófica, objetivo que no es desarrollado en este escrito. Agradezco el comentario de un/a revisor/a anónimo acerca de este punto.

13 Por ello previamente Machery (2017, cap. 2) descarta nociones como competencia conceptual o semántica.

14 El que ciertas intuiciones sean un insumo para la evaluación doxástica de cierto sujeto S, supone que se confía en las mismas en vistas a evaluar, por ejemplo, su variabilidad entre culturas. La inmodestia filosófica correspondería, en este caso, a una exigencia de restricción metodológica, utilizando insumos que se rechazan como confiables en un sentido general, las intuiciones en este caso. No hay en este sentido una distinción ad hoc entre que una fundamentación esté basada en intuiciones, o solo verse sobre intuiciones (queda por discutir qué consecuencias de implicación lógica tendría esta alternativa), pues la finalidad teórica es la misma en ambos casos: sostener la no confiabilidad en las intuiciones a partir del uso experimental de las mismas. Agradezco el comentario de un/a revisor/a anónimo en este punto. 